\title{
Comparisons of predictive values of sarcopenia with different muscle mass indices in Korean rural older adults: a longitudinal analysis of the Aging Study of PyeongChang Rural Area
}

This article was published in the following Dove Press journal:

Clinical Interventions in Aging

II-Young Jang, 1,2,* Hee-Won Jung, ${ }^{3,4, *}$ Chang Ki Lee, ${ }^{5}$ Sang Soo Yu, ${ }^{2}$ Young Soo Lee,' Eunju Lee $^{\prime}$

'Department of Internal Medicine, Asan Medical Center, University of Ulsan College of Medicine, Seoul, ${ }^{2}$ Pyeong Chang Health Center \& County Hospital, Gangwon-Do, ${ }^{3}$ Graduate School of Medical Science and Engineering, Korea Advanced Institute of Science and Technology (KAIST), Daejeon, ${ }^{4}$ Geriatric Center, Seoul National University Bundang Hospital, Gyeonggi-Do, ${ }^{5}$ Goldman Urology Clinic, Seoul, Republic of Korea

*These authors contributed equally to this work

Video abstract

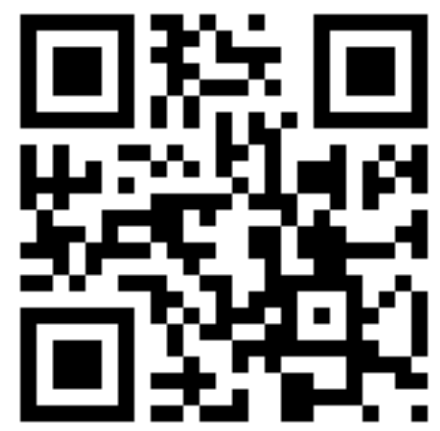

Point your SmartPhone at the code above. If you have a QR code reader the video abstract will appear. Or use:

http://youtu.be/bi8IWBiskY]

Correspondence: Eunju Lee

Department of Internal Medicine, Asan

Medical Center, University of Ulsan College

of Medicine, 88, Olympic-ro 43-gil, Songpa-gu,

Seoul 05505, Republic of Korea

Tel +82230103308

Fax +8224760824

Email eunjulee@amc.seoul.kr
Purpose: It is important to define lean muscle mass when diagnosing sarcopenia, but there is still controversy on the clinical implication of sarcopenia derived by height, weight, and body mass index (BMI) adjusted muscle mass indices. We aimed to evaluate the longitudinal clinical relevance of 3 sarcopenia definitions in the Korean population.

Patients and methods: We conducted comprehensive geriatric assessments for 1,379 community-dwelling older participants in the Aging Study of PyeongChang Rural Area (ASPRA) cohort and followed them up prospectively for death, institutionalization, and disability every 3 months. Sarcopenia was defined using the Asian Working Group consensus algorithm, combining grip strength, gait speed, and muscle mass.

Results: Among 1,343 participants (mean age: 76 years, 741 women) analyzed, there were 29 deaths and 89 institutionalizations during $22.0 \pm 8.3$ months follow-up (mean \pm SD). All three muscle indices correlated to age and sex. All sarcopenia criteria with muscle mass indices of height, weight, and body mass index (BMI) adjustment significantly predicted death or institutionalization. However, when adjusted for age and sex, only the sarcopenia criteria with muscle mass index of height adjustment were significantly associated with major adverse health outcomes.

Conclusion: Adjusting age and gender, the sarcopenia definition from the quintile-based muscle index of height adjustment could predict death or institutionalization in Korean communitydwelling older adults.

Keywords: cohort studies, frailty, geriatric assessment, muscle index, muscle mass, muscles, sarcopenia

\section{Introduction}

Sarcopenia is a common geriatric condition that is defined as a state of decreased muscle mass and performance with aging. This condition has been recognized as an independent clinical entity, of importance with regard to geriatric outcomes including mortality and functional decline, leading to its inclusion in the International Classification of Disease, Tenth Revision, Clinical Modification (ICD-10-CM) Code. ${ }^{1}$ In addition, with global population aging, the pharmaceutical industry ${ }^{2,3}$ and biomedical researchers ${ }^{4}$ are keen to elucidate treatable targets within the basic mechanisms of sarcopenia. Therefore, establishing clinically relevant criteria for sarcopenia in a specific population is more important than ever.

There have been numerous efforts to standardize the diagnostic algorithm of sarcopenia, including the monumental European consensus guideline ${ }^{5}$ and the consensus 
report from the Asian working group. ${ }^{6}$ In Korea, after a report that suggested Korean-specific cutoff values for weight- and height-adjusted appendicular skeletal muscle mass (ASM) from the Fourth Korean National Health and Nutritional Examination Surveys (KNHANES), ${ }^{7}$ studies have assessed for outcome relevance of various cutoff values and adjusting the method of muscle mass. ${ }^{8,9}$ However, with the birth cohort effect of the Korean population and the cultural background favoring leanness among young women, ASM appears to have an ageassociated increment in women. ${ }^{10}$ These controversies create a need for a sarcopenia definition that reflects age and sex.

Furthermore, many studies that assessed clinical features and outcome of sarcopenia in Korea choose muscle mass per se as an independent variable, rather than muscle strength or physical performance. ${ }^{11-13}$ Otherwise, they focused on a metabolic profile or metabolic consequences such as fatty liver diseases as outcomes, rather than geriatric outcomes including functional decline. ${ }^{11-13}$ Considering the characteristics of the Korean elderly population mentioned above, we hypothesized that we could find population-based cutoff points for each of the low-muscle-mass indices associated with clinical outcomes. Therefore, we aimed to evaluate the clinical relevance of various definitions of sarcopenia from the geriatric perspective, in a prospective cohort of the Aging Study of PyeongChang Rural Area (ASPRA) that is representative of general Korean rural-dwelling older people.

\section{Patients and methods}

\section{Study design and population}

The ASPRA is a population-based, prospective cohort study targeted to frailty and geriatric syndromes among communitydwelling older adults in Korean rural areas. Detailed design and methods pertaining to the ASPRA cohort are described elsewhere. ${ }^{14}$ Briefly, the study area is in PyeongChang County, Gangwon Province, South Korea, located 180 km east of Seoul. Inclusion criteria were 1) age 65 years or older; 2) registered in the National Healthcare Service; 3) ambulatory with or without an assistive device; 4) living at home; and 5) able to provide informed consent. Exclusion criteria were 1) living in a nursing home; 2) hospitalized; or 3 ) bedridden and receiving nursing home-level care at home at the time of enrollment. Between October 2014 and August 2017, 1,379 community-dwelling older adults participated in the ASPRA cohort, which included over $90 \%$ of eligible older adults living in the study area. ${ }^{14}$ All participants implemented a comprehensive geriatric assessment every 12 months, and health-related outcomes - including death, institutionalization, and disability - were recorded every 3 months.
A previous study showed that ASPRA participants have very similar characteristics to the nationally representative sample of South Korea - KNHANES (the Korea National Health and Nutrition Examination Survey) - except for the small difference in short educational period and higher agricultural workforce ratio. ${ }^{14}$ These characteristics ensure the appropriate generalizability of this population for descriptive figures that can represent Korean rural-dwelling older people. The Institutional Review Board of Asan Medical Center, Seoul, Korea, approved the protocol for this study, and all participants provided written informed consent.

\section{Measurements}

\section{Muscle mass}

To evaluate body composition including total mass and lean mass, we used bioelectrical impedance analysis (InBody 620; InBody, Seoul, Republic of Korea) with the use of measuring frequencies of 5, 50, and $500 \mathrm{kHz}$. The device measured the impedance of four limbs while participants were standing and calculated the skeletal muscle mass. To minimize interindividual differences, all participants were asked to fast overnight. ASM was calculated as the sum of the lean mass of both arms and legs provided from the manufacturer's algorithm. Previous studies have validated that bioelectrical impedance analysis is comparable to dual-energy X-ray absorptiometry. ${ }^{15}$

\section{Grip strength}

To assess the handgrip strength (kg), we used a dynamometer (T.K.K. 5401 Grip-D; Takei, Tokyo, Japan). ${ }^{16}$ Sitting comfortably with arm bent $90^{\circ}$ over the knee, participants were asked to hold the dynamometer as strongly as possible. ${ }^{17}$ All tests were conducted two times at intervals of 1 minute or more. The maximum value from the dominant arm was used for the analysis.

\section{Gait speed}

To evaluate the usual gait speed, participants were instructed to walk a total of 7 meters at a comfortable, usual, walking speed in a flat indoor space. Trained nurses measured the transit time of the 4-m distance from standing to the first footstep at the 4-m line by using a standard digital stopwatch and excluding an acceleration and deceleration interval of $1.5 \mathrm{~m}$, respectively. ${ }^{6}$ Finally, the usual gait speed $(\mathrm{m} / \mathrm{s})$ was calculated as the time taken to walk $4 \mathrm{~m}(\mathrm{~m} / \mathrm{s}) .{ }^{18}$

\section{Definition of sarcopenia}

To compare known several sarcopenia definitions, low muscle mass was defined as the sex-specific lowest quintile ${ }^{10}$ 
of ASM adjusted for height squared (ASM/ht $\left.{ }^{2}\right),{ }^{6}$ weight (ASM/wt), ${ }^{13}$ or BMI (ASM/BMI) ${ }^{19}$ respectively. We adopted the definition of low muscle mass using quintiles because the definition has been widely accepted by several sarcopenia definitions. ${ }^{5}$ Additionally, low muscle strength was defined as decreased handgrip strength of less than $26 \mathrm{~kg}$ for men and of less than $18 \mathrm{~kg}$ for women. ${ }^{6}$ Low physical performance was defined as slow gait of less than $0.8 \mathrm{~m} / \mathrm{s} .{ }^{6}$ By combining muscle mass, strength, and physical performance, we determined sarcopenic status by the consensus algorithm from the Asian Working Group for Sarcopenia. ${ }^{6}$

\section{Geriatric syndromes}

To evaluate the functional status and common geriatric conditions, a comprehensive geriatric assessment was administered annually by trained nurses. To evaluate disability, seven activities of daily living (ADL), including bathing, continence, dressing, eating, toileting, transferring, and washing face and hands, or 10 instrumental activities of daily living (IADL), including food preparation, household chores, going out a short distance, grooming, handling finances, laundry, managing own medications, shopping, transportation, and using a telephone, were interviewed. ${ }^{20}$ We assessed multimorbidity - defined as having 2 or more of the 11 physician-diagnosed illnesses including angina, arthritis, asthma, cancer, chronic lung disease, congestive heart failure, diabetes, heart attack, hypertension, kidney disease, and stroke. Depression was defined as a Korean version of the Center for Epidemiological Studies Depression (CES-D) scale greater than 20 points. ${ }^{21}$ Polypharmacy was defined as taking 5 or more prescription medications.

\section{Outcome measurements}

In this study, major adverse health outcomes have been defined as death or institutionalization. All participants were traced to minor check up every 3 months as well as a major follow-up every year. In interviews with participants or family members, the outcome was recorded when they were found to have died or had been institutionalized for longer than 3 months.

Functional impairment was evaluated by ADL and IADL assessment at baseline and follow-up. The occurrence of disability was defined as the absence of disability in baseline evaluation but disability in follow-up measurement.

\section{Statistical analysis}

Baseline participant characteristics were analyzed by the independent $t$-test for continuous variables and the $\chi^{2}$ test for categorical variables. Scatterplots and correlation analysis using linear regression models were conducted to evaluate the effect of age and sex on each muscle mass index. We used unadjusted or age- and multimorbidity-adjusted Cox proportional hazards models for men and women to clarify the clinical relevance of each muscle index on outcome, combining mortality and institutionalization due to functional decline. In addition, we used logistic regression analyses to evaluate whether sarcopenia criteria with each muscle mass index can predict functional impairment of ADL or IADL during follow-up periods. Statistical analyses were conducted using STATA 15.0 (StataCorp, College Station, TX, USA).

\section{Results \\ Baseline characteristics according to 3 sarcopenia definitions}

Among data from 1,379 ASPRA's participants, we excluded 36 people (14 men and 22 women) for missing data on bioelectrical impedance analysis, grip strength, or gait speed. Finally, 1,343 (602 men and 741 women) were analyzed for this study. The mean age of participants analyzed was 76.0 years (standard deviation [SD] 6.5 years), and the mean BMI was 24.6 (SD $3.4 \mathrm{~kg} / \mathrm{m}^{2}$ ). During the mean follow-up period of 22.0 months (SD 8.3 months), 29 participants died and 89 participants were hospitalized over 3 months.

Baseline characteristics of the participants according to the sarcopenia algorithms by 3 muscle mass indices are shown in Table 1. Briefly, participants with sarcopenia defined according to each muscle index were similar in age, sex, and duration of formal education. With sarcopenia, physical performance by SPPB score as well as frailty status were worse. Furthermore, the status of common geriatric syndromes including disability (both ADL and IADL), depressive mood, cognitive dysfunction, and polypharmacy was worse with the presence of sarcopenia.

\section{Classification according to sex-specific effects of appendicular skeletal muscle}

To assess the contribution of age and sex effects, we plotted the distribution of muscle mass indices calculated from three sarcopenia definitions (Figure 1). When adjusting sex in linear regression analysis, age was associated with each muscle index: 1) ASM $/ \mathrm{ht}^{2}$ : standardized beta $[\beta]=-0.267$, $P<0.001$; 2) ASM/wt: $\beta=-0.157, P=0.001$; and 3$) \mathrm{ASM} /$ BMI: $\beta=-0.201, P<0.001$.

Thereafter, we classified muscle indices into four categories by sex-specific quintiles. Quintile cutoff values of 
Table I Baseline characteristics according to three sarcopenia definitions

\begin{tabular}{|c|c|c|c|c|c|c|c|c|c|}
\hline \multirow[t]{3}{*}{ Characteristics } & \multicolumn{3}{|l|}{ ASM/ht ${ }^{2}$} & \multicolumn{3}{|l|}{ ASM/wt } & \multicolumn{3}{|l|}{ ASM/BMI } \\
\hline & \multirow{2}{*}{$\frac{\text { Non-sarcopenia }}{N=1,122}$} & \multirow{2}{*}{$\frac{\text { Sarcopenia }}{\mathrm{N}=221}$} & \multirow{2}{*}{$p$-value } & \multirow{2}{*}{$\frac{\text { Non-sarcopenia }}{\mathbf{N}=\text { I, I } 34}$} & \multirow{2}{*}{$\frac{\text { Sarcopenia }}{\mathrm{N}=\mathbf{2 0 9}}$} & \multirow{2}{*}{ p-value } & \multirow{2}{*}{$\frac{\text { Non-sarcopenia }}{N=1,128}$} & \multirow{2}{*}{$\frac{\text { Sarcopenia }}{\mathrm{N}=215}$} & \multirow[t]{2}{*}{$p$-value } \\
\hline & & & & & & & & & \\
\hline Age (mean, SD) & $75.0(6.0)$ & $81.1(6.4)$ & $<0.001$ & $75.3(6.3)$ & $80.0(6.3)$ & $<0.001$ & $75.1(6.1)$ & $80.8(6.3)$ & $<0.001$ \\
\hline Female (n, \%) & $604(53.8)$ & $137(62.0)$ & 0.031 & $614(54.1)$ & $127(60.8)$ & 0.090 & $608(53.9)$ & $133(61.9)$ & 0.038 \\
\hline $\begin{array}{l}\text { Education years } \\
\text { (mean, SD) }\end{array}$ & $5.4(3.6)$ & $4.0(2.0)$ & $<0.001$ & $5.4(3.6)$ & $4.2(2.4)$ & $<0.001$ & $5.4(3.6)$ & $4.1(2.1)$ & $<0.001$ \\
\hline Low income $(n, \%)$ & $64(5.7)$ & $21(9.5)$ & 0.049 & $66(5.8)$ & $19(9.1)$ & 0.103 & $68(6.0)$ & 17 (7.9) & 0.377 \\
\hline $\begin{array}{l}\text { Multimorbidity } \\
(\mathrm{n}, \%)\end{array}$ & 437 (38.9) & $94(42.5)$ & 0.357 & $4 I I(36.2)$ & $120(57.4)$ & $<0.001$ & $417(37.0)$ & $114(53.0)$ & $<0.001$ \\
\hline $\begin{array}{l}\text { K-FRAIL score } \\
\text { (mean, SD) }\end{array}$ & I.II (I.2) & $2.0(1.2)$ & $<0.001$ & I.I (I.2) & $2.0(1.1)$ & $<0.001$ & I.I (I.2) & $2.0(I . I)$ & $<0.001$ \\
\hline $\begin{array}{l}\text { CHS score } \\
\text { (mean, SD) }\end{array}$ & $1.7(1.23)$ & $2.6(1.0)$ & $<0.001$ & I.7 (I.3) & $2.5(1.0)$ & $<0.001$ & I.7 (I.2) & $2.6(1.0)$ & $<0.001$ \\
\hline ADL disability $(n, \%)$ & $122(10.9)$ & $49(22.2)$ & $<0.001$ & II7 (I0.3) & $54(25.8)$ & $<0.001$ & I24 (I I.0) & 47 (2I.9) & $<0.001$ \\
\hline $\begin{array}{l}\text { IADL disability } \\
(\mathrm{n}, \%)\end{array}$ & $303(27.0)$ & II 8 (53.4) & $<0.001$ & $306(27.0)$ & II 5 (55.0) & $<0.001$ & $307(27.2)$ & II 4 (53.0) & $<0.001$ \\
\hline Depression (n, \%) & $92(8.2)$ & $37(16.7)$ & $<0.001$ & $97(8.6)$ & $32(15.3)$ & 0.004 & $98(8.7)$ & $31(14.4)$ & 0.013 \\
\hline $\begin{array}{l}\text { Cognitive } \\
\text { dysfunction (n, \%) }\end{array}$ & $272(24.2)$ & $108(48.9)$ & $<0.001$ & $282(24.9)$ & $98(46.9)$ & $<0.001$ & $270(23.9)$ & $110(51.2)$ & $<0.001$ \\
\hline Polypharmacy (n, \%) & 235 (20.9) & $66(29.9)$ & 0.005 & $223(19.7)$ & $78(37.3)$ & $<0.001$ & $227(20.1)$ & $74(34.4)$ & $<0.001$ \\
\hline $\begin{array}{l}\text { Fall history for } \\
\text { previous I year } \\
\text { (mean, SD) }\end{array}$ & $0.2(0.8)$ & $0.4(\mathrm{I} .2)$ & 0.024 & $0.2(0.9)$ & $0.3(0.9)$ & 0.474 & $0.2(0.9)$ & $0.4(I . I)$ & 0.046 \\
\hline
\end{tabular}

Abbreviations: ADL, activities of daily living; ASM, appendicular skeletal muscle mass; BMI, body mass index; CHS, Cardiovascular Health Study; ht ${ }^{2}$, height square adjusted; IADL, instrumental activity of daily living; K-FRAIL, the Korean version of the Fatigue, Resistance, Ambulation, Illnesses, and Loss of weight questionnaire; SD, standard deviation; SPPB, short physical performance battery; wt, weight adjusted.

muscle indices adjusted by height, weight, and BMI are shown in Table 2.

\section{Major health adverse events by each sarcopenia definition}

To compare future major health adverse events by various muscle indices, we first compared each of the KaplanMeier curves of sarcopenia defined by the three muscle indices (Figure 2). Regardless of the adjusting method of muscle indices, the presence of sarcopenia was associated with significantly higher mortality and institutionalization events.

We investigated the risk of major health adverse events with the Cox proportional hazards model for men and women (Table 3). In an unadjusted model in men, the hazard ratios (HRs) for major health adverse events were 5.48 (95\% confidence interval [CI] 3.22-9.36) with sarcopenia criteria with muscle mass index of height adjustment, 3.32 (95\% CI 1.89-5.84) with muscle mass index of weight adjustment, and 3.84 (95\% CI 2.22-6.66) with muscle mass index of BMI adjustment. When adjusted for age and multimorbidity, sarcopenia criteria with muscle mass index of height adjustment still had the highest HR for major adverse health outcome (HR 5.18, 95\% CI 3.03-8.85). In an unadjusted model in women, the HRs for major health adverse events were 2.88 (95\% CI 2.26-4.75) with sarcopenia criteria with muscle mass index of height adjustment, 1.49 (95\% CI 0.83-2.66) with muscle mass index of weight adjustment, and 1.63 (95\% CI 0.94-2.85) with muscle mass index of BMI adjustment. When adjusted for age and multimorbidity, only sarcopenia criteria with muscle mass index of height adjustment was significantly associated with major adverse health outcome (HR 2.16, 95\% CI 1.28-3.65).

\section{Occurrence of functional impairment}

With regard to occurrences of functional impairment, logistic regression analysis was conducted, with the outcome as the newly developed ADL or IADL at the follow-up CGA. Unadjusted odds ratios for the occurrence of ADL disability were 1.65 (95\% CI 1.07-2.54) from sarcopenia criteria with height-adjusted muscle mass index, 1.68 (95\% CI 1.08-2.61) from the criteria with weight-adjusted muscle mass index, and 1.95 (95\% CI 1.28-2.97) from the criteria with BMI-adjusted muscle mass index, respectively. In a model adjusted for age and sex, the odds ratios for ADL disability from sarcopenia criteria with muscle mass indices of height, weight, and BMI adjustment were 1.30 (95\% CI 0.81-2.07), 1.42 (95\% CI $0.90-2.26$ ), and 1.65 (95\% CI 1.05-2.59), respectively. 

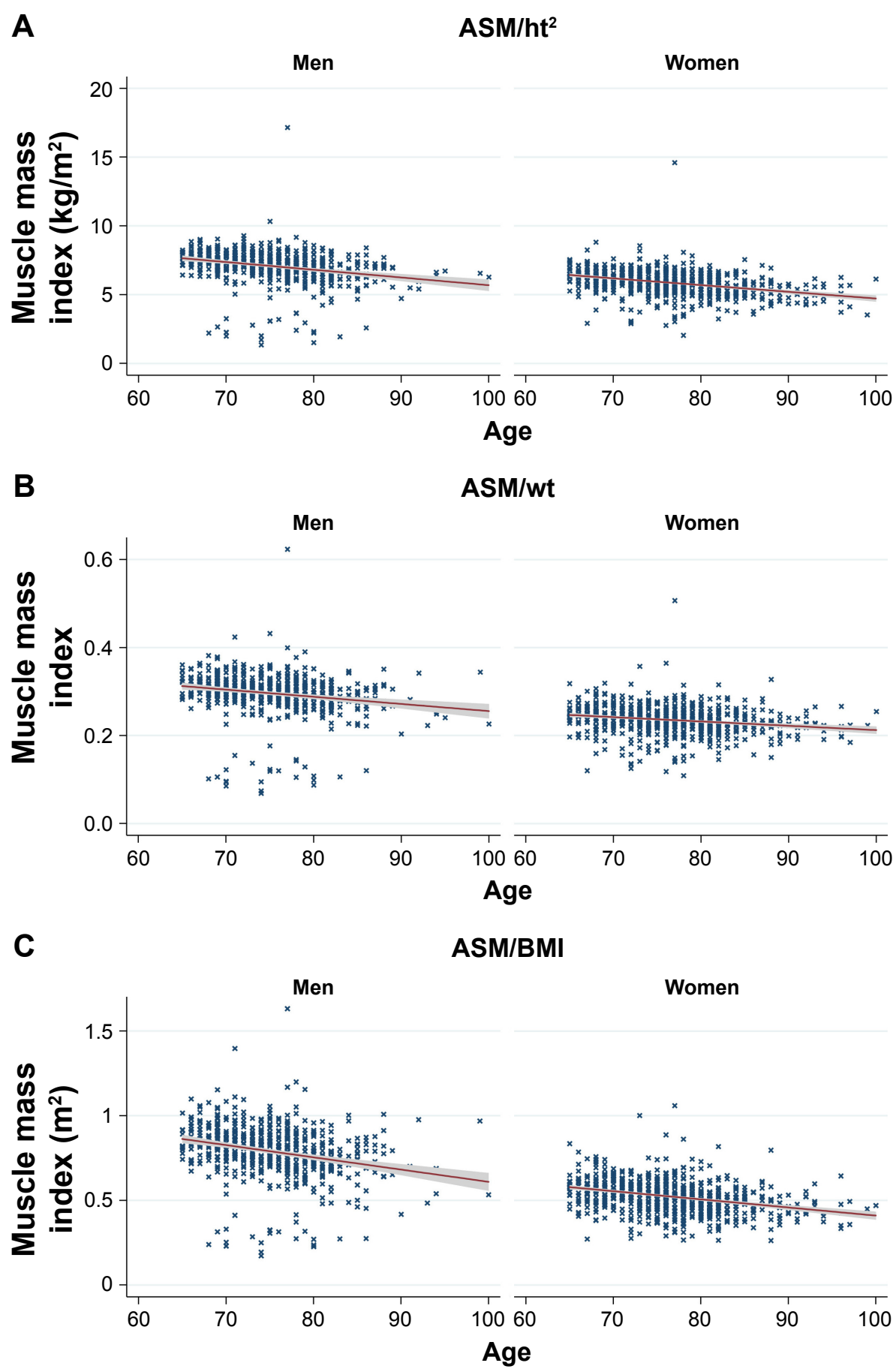

Figure I Sex-specific, age-dependent distributions of muscle indices; (A) height adjustment (ASM/ht'2), (B) weight adjustment (ASM/wt), and (C) BMI adjustment (ASM/BMI). Abbreviations: ASM, appendicular skeletal muscle mass; BMI, body mass index; ht', height square adjusted; wt, weight adjusted.

For the occurrence of IADL disability, the odds ratios were 2.97 (95\% CI 1.84-4.78) from sarcopenia criteria with muscle mass index of height adjustment, 3.28 (95\% CI 2.02-5.33) from the criteria with weight-adjusted muscle mass index, and $2.90(95 \%$ CI 1.80-4.65) from the criteria with BMI-adjusted muscle mass index, respectively. In an age- and sex-adjusted model, the odds ratios for IADL disability from sarcopenia criteria with muscle mass indices of height, weight, and BMI adjustment were 2.15 (95\% CI 1.30-3.57), 2.50 (95\% CI $1.51-4.15)$, and 2.08 (95\% CI 1.26-3.45), respectively.

\section{Discussion}

In this study, we compared major health adverse outcomes according to the three sarcopenia definitions in a 22-month 
Table 2 Sex-specific percentiles of appendicular muscle mass indices by height $\left(\mathrm{ASM} / \mathrm{ht}^{2}\right.$ ), weight (ASM/wt), and BMI (ASM/ $\mathrm{BMI})$ adjustment

\begin{tabular}{|c|c|c|c|c|c|c|c|c|}
\hline \multirow{3}{*}{$\begin{array}{l}\text { Muscle } \\
\text { mass } \\
\text { index }\end{array}$} & \multicolumn{8}{|c|}{ Percentiles } \\
\hline & \multicolumn{4}{|l|}{ Men } & \multicolumn{4}{|c|}{ Women } \\
\hline & 20 & 40 & 60 & 80 & 20 & 40 & 60 & 80 \\
\hline $\begin{array}{l}\text { ASM } / \mathrm{ht}^{2} \\
\left(\mathrm{~kg} / \mathrm{m}^{2}\right)\end{array}$ & 6.439 & 7.014 & 7.459 & 7.917 & 5.197 & 5.702 & 6.118 & 6.571 \\
\hline $\begin{array}{l}\text { ASM/wt } \\
(\%)\end{array}$ & 27.547 & 29.292 & 30.794 & 32.522 & 21.383 & 22.936 & 24.309 & 25.883 \\
\hline $\begin{array}{l}\text { ASM/BMI } \\
\left(\mathrm{m}^{2}\right)\end{array}$ & 0.693 & 0.766 & 0.832 & 0.906 & 0.446 & 0.499 & 0.541 & 0.598 \\
\hline
\end{tabular}

Abbreviations: ASM, appendicular skeletal muscle; BMI, body mass index; $\mathrm{ht}^{2}$, height square adjusted; wt, weight adjusted.

follow-up of a rural community-dwelling cohort potentially representative of general rural-dwelling older adults of South Korea. We observed sex-specific distributions of muscle indices adjusted by height, weight, and BMI. Moreover, when combined with physical performances of gait speed and grip strength, sarcopenia definitions using all three of these adjustment methods for muscle indices had significant hazard risks of major health adverse events in death or institutionalization in men, even after age and multimorbidity adjustment. For women, only sarcopenia defined using muscle mass index of height adjustment was associated with significantly increased risk for major health outcomes before and after age and multimorbidity adjustment.

Ethnic differences in anthropometric parameters and outcome relevance have caused researchers to establish population-specific definitions of the decreased lean muscle mass in many countries. Our cutoff points using the lower quintile were $<6.439 \mathrm{~kg} / \mathrm{m}^{2}$ for men and $<5.197 \mathrm{~kg} / \mathrm{m}^{2}$ for women in height-adjusted muscle mass index, and $<0.693 \mathrm{~m}^{2}$ for men and $<0.446$ for women in the BMI-adjusted muscle mass index, and comparable to reports from other countries. Considering the height-adjusted muscle mass index, bioelectrical analysis (BIA)-using cutoff points for decreased muscle mass defined as 2SD lower than healthy young adults were reported as $<8.87 \mathrm{~kg} / \mathrm{m}^{2}$ for men and $<6.42 \mathrm{~kg} / \mathrm{m}^{2}$ for
A

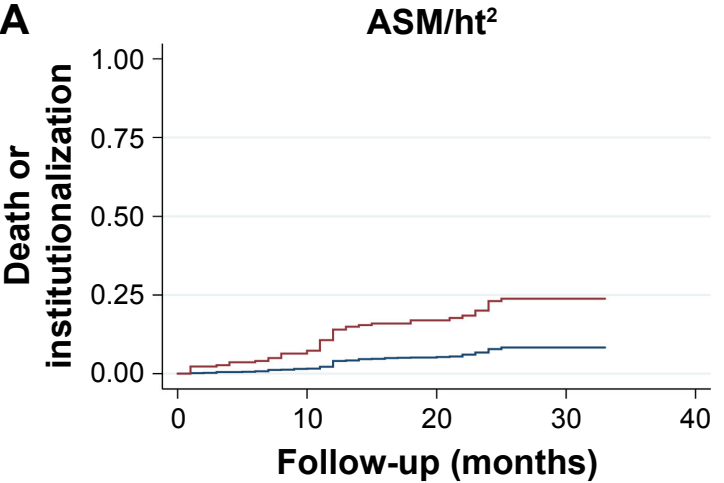

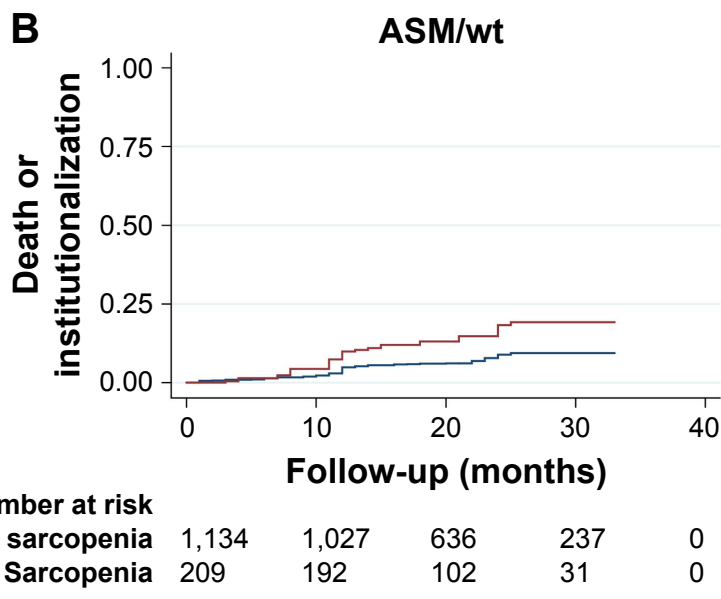

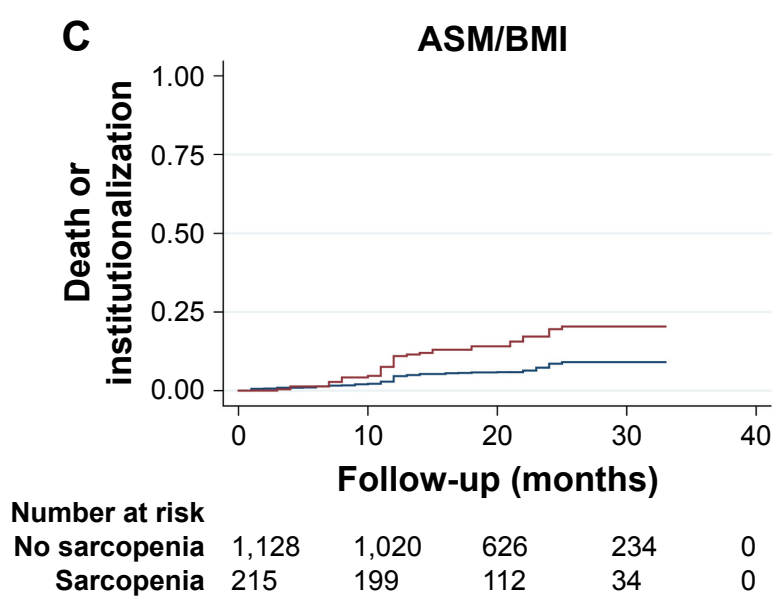

Figure 2 Kaplan-Meier curves showing death or institutionalization during follow-up, with regard to sarcopenia status with various muscle indices: (A) height adjustment (ASM/ht ${ }^{2}$ ), (B) weight adjustment (ASM/wt), and (C) BMI adjustment (ASM/BMI).

Note: Red lines: sarcopenia; blue lines: no sarcopenia.

Abbreviations: ASM, appendicular skeletal muscle; BMI, body mass index; $\mathrm{ht}^{2}$, height square adjusted; wt, weight adjusted. 
Table 3 Survival analysis using Cox proportional hazard models illustrating the effect of sarcopenia status with various muscle indices on the combined outcome of mortality and institutionalization during follow-up period

\begin{tabular}{|c|c|c|c|}
\hline Model & HR & $95 \% \mathrm{Cl}$ & $p$-value \\
\hline \multicolumn{4}{|l|}{ Men } \\
\hline Unadjusted & 1.00 & & \\
\hline Sarcopenia-ASM/ht ${ }^{2}$ & 5.48 & $3.22-9.36$ & $<0.001$ \\
\hline Sarcopenia-ASM/wt & 3.32 & I.89-5.84 & $<0.001$ \\
\hline Sarcopenia-ASM/BMI & 3.84 & $2.22-6.66$ & $<0.00 \mathrm{I}$ \\
\hline Age, multimorbidity adjusted & 1.00 & & \\
\hline Sarcopenia-ASM $/ \mathrm{ht}^{2}$ & 5.18 & $3.03-8.85$ & $<0.001$ \\
\hline Sarcopenia-ASM/wt & 1.77 & $1.02-3.08$ & 0.001 \\
\hline Sarcopenia-ASM/BMI & 3.33 & I.89-5.88 & $<0.001$ \\
\hline \multicolumn{4}{|l|}{ Women } \\
\hline Unadjusted & 1.00 & & \\
\hline Sarcopenia-ASM/ht ${ }^{2}$ & 2.88 & $2.26-4.75$ & 0.004 \\
\hline Sarcopenia-ASM/wt & 1.49 & $0.83-2.66$ & 0.179 \\
\hline Sarcopenia-ASM/BMI & 1.63 & $0.94-2.85$ & 0.083 \\
\hline Age, multimorbidity adjusted & 1.00 & & \\
\hline Sarcopenia-ASM/ht ${ }^{2}$ & 2.16 & $1.28-3.65$ & 0.004 \\
\hline Sarcopenia-ASM/wt & 1.48 & $0.83-2.67$ & 0.183 \\
\hline Sarcopenia-ASM/BMI & 1.63 & $0.93-2.85$ & 0.085 \\
\hline
\end{tabular}

Abbreviations: ASM, appendicular skeletal muscle mass; BMI, body mass index; $\mathrm{Cl}$, confidence interval; HR, hazard ratio; $h^{2}$, height square adjusted; wt, weight adjusted.

women in Taiwan, ${ }^{22}<7.0 \mathrm{~kg} / \mathrm{m}^{2}$ for men and $<5.8 \mathrm{~kg} / \mathrm{m}^{2}$ for women in Japan, ${ }^{23}<5.72 \mathrm{~kg} / \mathrm{m}^{2}$ for men and $<4.82 \mathrm{~kg} / \mathrm{m}^{2}$ forwomeninChina, ${ }^{24}$ and $<6.75 \mathrm{~kg} / \mathrm{m}^{2}$ formenand $<5.07 \mathrm{~kg} / \mathrm{m}^{2}$ for women in another report from Korea. ${ }^{25}$ In a study that used the lowest quintile of the muscle mass index for the definition of decreased muscle mass, cutoff points were $<7.27 \mathrm{~kg} / \mathrm{m}^{2}$ for men and $<5.46 \mathrm{~kg} / \mathrm{m}^{2}$ for women in Taiwan. ${ }^{26}$ With regard to the muscle index adjusted by BMI, our cutoff points were $0.693 \mathrm{~m}^{2}$ for men and $0.466 \mathrm{~m}^{2}$ for women, compared to $0.789 \mathrm{~m}^{2}$ for men and $0.512 \mathrm{~m}^{2}$ for women from the original report of the FNIH sarcopenia project. ${ }^{19}$ Cut-off points from the ASPRA population were relatively lower than other studies, of which these differences are probably due to the high participation rate of the ASPRA cohort with lower selection bias from including more frail participants from among community-dwelling older adults.

Varying definitions of sarcopenia have been proposed by several authoritative research groups in order to diagnose sarcopenia. In a long controversy, how to define the reduced skeletal muscle mass was an important issue. In the past, sarcopenia was defined as a state of decreased muscle mass, such as a reduction of ASM of less than 2 SDs from the healthy young adult population, or the lowest quintile value in the study population. ${ }^{27-29}$ However, since muscle strength or physical performance is more important in predicting health outcome than ASM alone, ${ }^{30}$ the sarcopenia criteria - defined as a combination of muscle mass with slowness and/or weakness - proposed by the European working group and the Asian group, has been widely applied. ${ }^{5,6}$ Recently, moreover, the Sarcopenia Project of the National Institutes of Health (FNIH) group has defined ASM/BMI as low lean mass following analysis of nine large community-based cohorts. ${ }^{19}$ They primarily defined lean muscle mass in relation to body composition and mobility limitation. However, various definitions of sarcopenia, discussed above, have been reported in different clinical implications, depending on the population, age, socioeconomic status, or type of outcome. ${ }^{10,31-33}$ For possible differences of muscle mass, strength, and physical performance pertaining to specific populations, we should adopt cutoff values and adjustment methods of muscle mass indices and definitions of sarcopenia in a population-specific manner. ${ }^{34,35}$

There have been several reports of FNIH-defined sarcopenia criteria predicting mortality or cognitive decline in Koreans. ${ }^{36,37}$ Recently, FNIH-defined sarcopenia criteria have been reported to predict death and adverse events from I-Lan Longitudinal Aging Study in Taiwan. ${ }^{38}$ However, we showed that only a sarcopenia definition with height-adjusted muscle index was significantly associated with death or institutionalization, rather than FNIH sarcopenia criteria when adjusted for age and sex. This difference may be due to heterogeneities in the characteristics of the study population, outcome measures, and the duration of follow-up. In contrast, with our secondary analysis for functional decline, the FNIH definition consistently predicted the occurrence of ADL or IADL disability even after adjusting for age and sex. This similarity is probably due to the fact that the FNIH definition is made with the mobility limitation as a key outcome.

Our study has several strengths and limitations. First, the ASPRA cohort is based on the Korean older population living in rural communities. However, the demographic characteristics of ASPRA share similarities with the national representative sample - KNHANES - and has a high participation rate of eligible older adults in the research area. ${ }^{14}$ All measurement tools in ASPRA cohort are well standardized and cover most geriatric syndromes. Second, for measurement of muscle mass, this study used bioelectrical impedance analysis which is known to be very accurate and reproducible in evaluating skeletal muscle mass, and the standard method for sarcopenia diagnosis by the authorized guideline. ${ }^{6,39}$ Third, the population-based ASPRA cohort does not include laboratory or biomarker findings. Nevertheless, as in previous cardiology studies of gait speed and clinical outcome, ${ }^{40,41}$ 
clinical indices have been reported to be as relevant as the results of metabolic profiles composed of laboratory results. Rather, this non-invasiveness also leads to a high retention rate per year of approximately $90 \%$ or more to increase the value of the study.

\section{Conclusion}

From this study, we provide distributions of muscle mass indices that can be used in algorithms of sarcopenia, in a nationwide representative rural-dwelling older population of Korea for the first time. Moreover, we found that a sarcopenia definition which combined grip strength and gait speed with muscle mass index adjusted for height is most highly associated with major adverse health outcome when adjusted for age and multimorbidity in both men and women.

\section{Acknowledgments}

We are indebted to public health professionals and nurses of PyeongChang County Hospital, Public Health Center, and Community Health Posts for their administrative support and help in enrollment, retention, and measurements. We especially appreciate Hyelim Park, Seon-hee Cheon, Chawoong Jeon, and Jae Kyoon Hwang from PyeongChang County Hospital.

The Aging Study of PyeongChang Rural Area was supported by PyeongChang County Hospital, PyeongChang County, Gangwon Province, Korea. The present study was supported, in part, by Paul Park and Maeil Dairies Co., Ltd. from the Asan Institute for Life Sciences and Corporate Relations of the Asan Medical Center, Seoul, Republic of Korea.

Dr Hee-Won Jung was supported by a Global PhD Fellowship Program through the National Research Foundation of Korea funded by the Ministry of Education (NRF2015H1A2A1030117).

Public health professionals and nurses of PyeongChang County Hospital were involved in data collection, but they did not have any role in the study design, analysis or interpretation of data, in the writing of the report, or in the decision to submit it for publication. Paul Park and Maeil Dairies Co., Ltd. did not have any role in the study design, in the collection, analysis or interpretation of data, in the writing of the report, or in the decision to submit it for publication.

\section{Author contributions}

All authors participated in designing the study concept, undertook statistical analysis, data analysis, and interpretation, and drafted the manuscript. They agree to be accountable for all aspects of the work in ensuring that the accuracy or integrity of any part of the work is appropriately investigated and resolved. All authors have read and revised this manuscript. This manuscript is the final approved version from all authors. All authors have reviewed and agreed to be responsible for the process, accuracy, and integrity of all parts of this manuscript.

\section{Disclosure}

The authors report no conflicts of interest in this work.

\section{References}

1. Cao L, Morley JE. Sarcopenia is recognized as an independent condition by an International Classification of Disease, Tenth Revision, Clinical Modification (ICD-10-CM) Code. J Am Med Dir Assoc. 2016; 17(8):675-677.

2. Becker C, Lord SR, Studenski SA, et al; STEADY Group. Myostatin antibody (LY2495655) in older weak fallers: a proof-of-concept, randomised, phase 2 trial. Lancet Diabetes Endocrinol. 2015;3(12): 948-957.

3. Cohen S, Nathan JA, Goldberg AL. Muscle wasting in disease: molecular mechanisms and promising therapies. Nat Rev Drug Discov. 2015; 14(1):58-74.

4. Benoit B, Meugnier E, Castelli M, et al. Fibroblast growth factor 19 regulates skeletal muscle mass and ameliorates muscle wasting in mice. Nat Med. 2017;23(8):990-996.

5. Cruz-Jentoft AJ, Baeyens JP, Bauer JM, et al; European Working Group on Sarcopenia in Older People. Sarcopenia: European consensus on definition and diagnosis: Report of the European Working Group on Sarcopenia in Older People. Age Ageing. 2010;39(4):412-423.

6. Chen LK, Liu LK, Woo J, et al. Sarcopenia in Asia: consensus report of the asian working group for sarcopenia. J Am Med Dir Assoc. 2014;15(2): 95-101.

7. Kim YS, Lee Y, Chung YS, et al. Prevalence of sarcopenia and sarcopenic obesity in the Korean population based on the Fourth Korean National Health and Nutritional Examination Surveys. J Gerontol A Biol Sci Med Sci. 2012;67(10):1107-1113.

8. Kim JH, Lim S, Choi SH, et al. Sarcopenia: an independent predictor of mortality in community-dwelling older Korean men. $J$ Gerontol A Biol Sci Med Sci. 2014;69(10):1244-1252.

9. Jung HW, Kim SW, Chin HJ, Kim KI, Kim CH. Skeletal muscle mass as a predictor of mortality in the elderly population. J Korean Med Assoc. 2013;85(2):167-173.

10. Chang EJ, Jung HW, Kim SW, et al. Determining the cut-off values for sarcopenia in the Korean elderly population using bioimpedance analysis. J Frailty Aging. 2015;4(1):34-40.

11. Lee Yh, Jung KS, Kim SU, et al. Sarcopaenia is associated with NAFLD independently of obesity and insulin resistance: Nationwide Surveys (KNHANES 2008-2011). J Hepatol. 2015;63(2):486-493.

12. Kim SW, Jung HW. Which one is associated with nonalcoholic fatty liver disease? Small muscle mass or large fat mass. Hepatology. 2015; 61(5): 1764.

13. Kim KM, Jang HC, Lim S. Differences among skeletal muscle mass indices derived from height-, weight-, and body mass index-adjusted models in assessing sarcopenia. Korean J Intern Med. 2016;31(4):643-650.

14. Jung HW, Jang IY, Lee YS, et al. Prevalence of frailty and aging-related health conditions in older Koreans in rural communities: a cross-sectional analysis of the Aging Study of Pyeongchang Rural Area. J Korean Med Sci. 2016;31(3):345-352.

15. Kim M, Shinkai S, Murayama H, Mori S. Comparison of segmental multifrequency bioelectrical impedance analysis with dual-energy X-ray absorptiometry for the assessment of body composition in a community-dwelling older population. Geriatr Gerontol Int. 2015;15(8): 1013-1022. 
16. Hachiya M, Murata S, Otao H, Kamijou K, Mizota K, Asami T. Reproducibility and validity of the 50-meter walking test in communitydwelling elderly. J Phys Ther Sci. 2015;27(5):1511-1514.

17. Mathiowetz V, Rennells C, Donahoe L. Effect of elbow position on grip and key pinch strength. J Hand Surg Am. 1985;10(5):694-697.

18. Peters DM, Fritz SL, Krotish DE. Assessing the reliability and validity of a shorter walk test compared with the 10-Meter Walk Test for measurements of gait speed in healthy, older adults. J Geriatr Phys Ther. 2013; 36(1):24-30.

19. Studenski SA, Peters KW, Alley DE, et al. The FNIH sarcopenia project: rationale, study description, conference recommendations, and final estimates. J Gerontol A Biol Sci Med Sci. 2014;69(5):547-558.

20. Won CW, Yang KY, Rho YG, et al. The development of Korean Activities of Daily Living (K-ADL) and Korean Instrumental Activities of Daily Living (K-IADL) Scale. J Korean Geriatr Soc. 2002;6(2): 107-120

21. Park JH, Kim KW. A review of the epidemiology of depression in Korea. J Korean Med Assoc. 2011;54(4):362-369.

22. Nigam P, Misra A, Colles SL. Comparison of DEXA-derived body fat measurement to two race-specific bioelectrical impedance equations in healthy Indians. Diabetes Metab Syndr. 2013;7(2):72-77.

23. Tanimoto Y, Watanabe M, Sun W, et al. Association between sarcopenia and higher-level functional capacity in daily living in communitydwelling elderly subjects in Japan. Arch Gerontol Geriatr. 2012;55(2): e9-e13.

24. Lau EM, Lynn HS, Woo JW, Kwok TC, Melton LJ 3rd. Prevalence of and risk factors for sarcopenia in elderly Chinese men and women. J Gerontol A Biol Sci Med Sci. 2005;60(2):213-216.

25. Goodpaster BH, Park SW, Harris TB, et al. The loss of skeletal muscle strength, mass, and quality in older adults: the health, aging and body composition study. J Gerontol A Biol Sci Med Sci. 2006;61(10): 1059-1064.

26. Lee WJ, Liu LK, Peng LN, Lin MH, Chen LK. Comparisons of sarcopenia defined by IWGS and EWGSOP criteria among older people: results from the I-Lan longitudinal aging study. J Am Med Dir Assoc. 2013;14(7):528.e521-528.e527.

27. Baumgartner RN, Koehler KM, Gallagher D, et al. Epidemiology of sarcopenia among the elderly in New Mexico. Am J Epidemiol. 1998 147(8):755-763.

28. Fielding RA, Vellas B, Evans WJ, et al. Sarcopenia: an undiagnosed condition in older adults. Current consensus definition: prevalence, etiology, and consequences. International working group on sarcopenia. J Am Med Dir Assoc. 2011;12(4):249-256.
29. Newman AB, Kupelian V, Visser M, et al. Sarcopenia: alternative definitions and associations with lower extremity function. J Am Geriatr Soc. 2003;51(11):1602-1609.

30. Newman AB, Kupelian V, Visser M, et al. Strength, but not muscle mass, is associated with mortality in the health, aging and body composition study cohort. J Gerontol A Biol Sci Med Sci. 2006;61(1):72-77.

31. Woo J, Leung J, Sham A, Kwok T. Defining sarcopenia in terms of risk of physical limitations: a 5-year follow-up study of 3,153 chinese men and women. J Am Geriatr Soc. 2009;57(12):2224-2231.

32. Cawthon PM, Blackwell TL, Cauley J, et al. Evaluation of the usefulness of consensus definitions of sarcopenia in older men: results from the observational osteoporotic fractures in men cohort study. J Am Geriatr Soc. 2015;63(11):2247-2259.

33. Jang IY, Jung HW, Lee CK, et al. Rural and urban disparities in frailty and aging-related health conditions in Korea. J Am Geriatr Soc. 2016; 64(4):908-911.

34. Woo J, Leung J. Anthropometric cut points for definition of sarcopenia based on incident mobility and physical limitation in older Chinese people. J Gerontol A Biol Sci Med Sci. 2016;71(7):935-940.

35. Woo J, Arai H, Ng TP, et al. Ethnic and geographic variations in muscle mass, muscle strength and physical performance measures. Eur Geriatr Med. 2014;5(3):155-164.

36. Moon JH, Kim KM, Kim JH, et al. Predictive values of the new sarcopenia index by the Foundation for the National Institutes of Health Sarcopenia Project for mortality among older Korean adults. PLoS One. 2016; 11(11):e0166344.

37. Moon JH, Moon JH, Kim KM, et al. Sarcopenia as a predictor of future cognitive impairment in older adults. J Nutr Health Aging. 2016; 20(5):496-502.

38. Tang TC, Hwang AC, Liu LK, et al. FNIH-defined sarcopenia predicts adverse outcomes among community-dwelling older people in Taiwan: results from I-Lan longitudinal aging study. J Gerontol A Biol Sci Med Sci. Epub 2017 Jul 25.

39. Janssen I, Heymsfield SB, Baumgartner RN, Ross R. Estimation of skeletal muscle mass by bioelectrical impedance analysis. J Appl Physiol (1985). 2000;89(2):465-471.

40. Pulignano G, Del Sindaco D, Di Lenarda A, et al; IMAGE-HF Study Investigators. Incremental value of gait speed in predicting prognosis of older adults with heart failure: insights from the IMAGE-HF study. JACC Heart Fail. 2016;4(4):289-298.

41. Afilalo J, Eisenberg MJ, Morin JF, et al. Gait speed as an incremental predictor of mortality and major morbidity in elderly patients undergoing cardiac surgery. J Am Coll Cardiol. 2010;56(20):1668-1676.
Clinical Interventions in Aging

\section{Publish your work in this journal}

Clinical Interventions in Aging is an international, peer-reviewed journal focusing on evidence-based reports on the value or lack thereof of treatments intended to prevent or delay the onset of maladaptive correlates of aging in human beings. This journal is indexed on PubMed Central, MedLine,

\section{Dovepress}

CAS, Scopus and the Elsevier Bibliographic databases. The manuscript management system is completely online and includes a very quick and fair peer-review system, which is all easy to use. Visit http://www.dovepress. com/testimonials.php to read real quotes from published authors. 